\title{
Glucose Uptake in Rat Skeletal Muscle L6 Cells Is Increased by Low-Intensity Electrical Current Through the Activation of the Phosphatidylinositol-3-OH Kinase (PI-3K) / Akt Pathway
}

\author{
Shuichiro Yano ${ }^{1}$, Saori Morino-Koga ${ }^{1}$, Tatsuya Kondo ${ }^{2}$, Mary Ann Suico ${ }^{1}$, Tomoaki Koga ${ }^{1}$, \\ Yuichiro Shimauchi ${ }^{1}$, Shingo Matsuyama ${ }^{1}$, Tsuyoshi Shuto ${ }^{1}$, Takashi Sato ${ }^{1}$, Eiichi Araki ${ }^{2}$, \\ and Hirofumi $\mathrm{Kai}^{1, *}$ \\ ${ }^{1}$ Department of Molecular Medicine, Graduate School of Pharmaceutical Sciences, \\ Global COE "Cell Fate Regulation Research and Education Unit”, Kumamoto University, Kumamoto 862-0973, Japan \\ ${ }^{2}$ Department of Metabolic Medicine, Faculty of Life Sciences, Kumamoto University, Kumamoto 860-8556, Japan
}

Received July 14, 2010; Accepted November 11, 2010

\begin{abstract}
Activation of Akt by insulin is transmitted via phosphatidylinositol-3-OH kinase (PI$3 \mathrm{~K}$ ) and enhances glucose uptake. The PI-3K/Akt signaling is diminished in insulin resistance. Thus, approaches that activate PI-3K/Akt signaling leading to improved glucose uptake may ameliorate hyperglycemia. Here we showed that low-intensity electrical current or mild electrical stimulation (MES) activated the PI-3K/Akt signaling and increased the glucose uptake in rat skeletal muscle (L6) cells. The glucose uptake enhanced by MES in muscle cells, the major cells involved in glucose disposal, suggests MES may have a possible beneficial effect on hyperglycemia.
\end{abstract}

Keywords: mild electrical stimulation, phosphatidylinositol-3-OH kinase (PI-3K) / Akt, glucose uptake

Insulin signaling regulates glucose uptake into muscle and fat cells by activating the phosphatidylinositol-3-OH kinase (PI-3K) / Akt pathway and inducing the translocation of glucose transporter (GLUT) 4 from intracellular storage to the plasma membrane $(1,2)$. Reduced sensitivity to insulin, which is commonly observed in obese diabetic patients, is associated with impaired PI-3K/Akt activity that ultimately results in the hyperglycemic condition (3). Thus, approaches that enhance PI-3K/Akt activation independently of insulin may be helpful in ameliorating hyperglycemia. We previously indicated that low-intensity electrical current or mild electrical stimulation (MES) in combination with mild heat shock $\left(42^{\circ} \mathrm{C}\right)$ alleviated insulin resistance and improved fat metabolism in mouse models of type 2 diabetes, in part, by enhancing the insulin-signaling pathway (4). Results of our preliminary investigation in healthy human subjects showed that MES with mild heat shock safely re-

*Corresponding author. hirokai@gpo.kumamoto-u.ac.jp Published online in J-STAGE on December 18, 2010 (in advance) doi: $10.1254 /$ jphs.10185SC duced the inflammatory markers independent of their body weight alterations (5). In our previous study, we found that one of the main target tissues of MES treatment is the liver, as suggested by the findings that lowintensity electrical current or MES [1 V/cm, 0.1 millisecond (ms), 55 pulses per second (pps)] increased the insulin-stimulated phosphorylation of Akt in hepatocytes (HepG2 cells) (4). Here, we hypothesize that other insulin target cells, such as skeletal muscle cells, might also be affected by MES treatment, and are involved in the observed amelioration of the diabetic phenotype. Because the skeletal muscle is a major tissue involved in the uptake of glucose, accounting for $75 \%$ of whole body glucose uptake (6), we investigated whether MES can induce $\mathrm{PI}-3 \mathrm{~K} / \mathrm{Akt}$ activation in muscle cells.

We used here rat skeletal muscle (L6) cells, a myogenic cell line that is commonly used for the investigation of the PI-3K/Akt signaling pathway in skeletal muscles. These cells were obtained from the American Type Culture Collection (Manassas, VA, USA). L6 cells were cultured in Dulbecco's modified Eagle's medium (DMEM) (Wako Pure Chemical Industries, Ltd., Osaka) 
containing $10 \%$ fetal bovine serum (FBS) and antibiotics and maintained in a $37^{\circ} \mathrm{C}$ incubator. Cells $\left(1 \times 10^{6}\right)$ were plated on $60-\mathrm{mm}$ culture dishes, and at $80 \%$ confluence, they were differentiated by culturing for 6 to 7 days in DMEM medium containing 2\% FBS. Medium was changed every other day. After differentiation, cells were treated for $10 \mathrm{~min}$ with MES at $0.01-10 \mathrm{~ms}, 5 \mathrm{~V}, 55 \mathrm{pps}$ or at $0.1 \mathrm{~ms}, 2-10 \mathrm{~V}, 55 \mathrm{pps}$. During treatment, the culture plate cover was exchanged with a plate cover slitted at the sides designed to accommodate insulated wires bearing a pair of flat rubber electrodes, which were fitted at the walls of the culture plate. The electrodes were connected to a BioMetronome ${ }^{\mathrm{TM}}$ (Tsuchiya Gum Co., Ltd., Kumamoto) as described previously $(4,7)$. Five minutes after treatment, cell lysates were extracted using lysis buffer and analyzed by Western blotting with anti-phosphorylated Akt and anti-Akt antibodies according to the previously described protocol (4). Interestingly, the data showed that MES activated Akt, and that $0.1 \mathrm{~ms}$ is the most effective pulse duration and $5 \mathrm{~V}$ (approximately $1 \mathrm{~V} / \mathrm{cm}$ ) is the minimum voltage of MES that induced the phosphorylation of Akt (Fig. 1: A and B). To determine how long MES could sustain the activation of Akt, differentiated L6 cells were treated with MES ( $1 \mathrm{~V} /$ $\mathrm{cm}, 0.1 \mathrm{~ms}$, and $55 \mathrm{pps}$ ) for $10 \mathrm{~min}$, and lysates were isolated at the indicated time points using lysis buffer. The effect of MES on Akt activation was highest immediately after treatment and slightly declined after $1 \mathrm{~h}$ (Fig. 1C), returning to basal level at $2 \mathrm{~h}$ (data not shown). Previous reports showed that electrical stimulation activates AMPK soon after $(<1 \mathrm{~h})$ treatment $(8,9)$; therefore, we also checked the phosphorylated form of AMPK after treatment with MES. Although MES activated Akt, it did not activate AMPK within this time span (Fig. 1C, pAMPK). The difference between our result and previous studies may be due to the condition of electrical current used. While in other reports the authors utilized strong electrical stimulation with supramaximal voltage (approximately $100 \mathrm{~V}$ or $10 \mathrm{~V} / \mathrm{cm})(8,9)$, here we used low-intensity electrical current (approximately $1 \mathrm{~V} / \mathrm{cm}$ ). This may account for the different observations on AMPK. Because MES treatment $(1 \mathrm{~V} / \mathrm{cm}, 0.1 \mathrm{~ms}, 55$ pps) for $10 \mathrm{~min}$ also induced Akt phosphorylation in differentiated L6 cells in the absence of FBS (Fig. 1D), serum growth factors might not be involved in the observed effect of MES on Akt activation.

To find out whether the effect of MES on the Akt pathway is due to muscle contraction, we pre-treated L6 cells with the muscle relaxant dantrolene $(25 \mu \mathrm{M})$ and then co-treated with MES for $10 \mathrm{~min}$. The Akt phosphorylation induced by MES was not affected by dantrolene (Fig. 1E). To support this observation, we treated L6 cells for 10 min with MES or with increasing concentra- tions of $\mathrm{KCl}(40-120 \mathrm{mM})$. Specifically, differentiated L6 cells were incubated in HEPES-buffered saline containing $5 \mathrm{mM}$ D-glucose, $20 \mathrm{mM}$ HEPES, $1 \mathrm{mM} \mathrm{CaCl}_{2}$, $2.5 \mathrm{mM} \mathrm{MgSO}_{4}$, and $\mathrm{NaCl}$. The concentration of $\mathrm{NaCl}$ was adjusted to maintain osmolarity. High $\mathrm{K}^{+}$concentration, which is known to induce muscle contraction, did not increase Akt phosphorylation, although it slightly induced the phosphorylation of AMPK (Fig. 1F). In addition, we evaluated the effect of MES on cell death. We found that MES at 5 and $10 \mathrm{~V}(1$ and $1.5 \mathrm{~V} / \mathrm{cm}$, respectively) did not induce LDH release (Fig. 1G). Moreover, MES did not decrease the mitochondrial membrane potential $(\Delta \psi \mathrm{m})($ Fig. $1 \mathrm{H})$ and did not induce morphological changes in the cells (data not shown). These data imply a lack of cytotoxicity of MES treatment.

To assess the effect of MES on the activation of PI-3K in muscle cells, L6 cells grown and differentiated on cover glasses were treated with MES for $10 \mathrm{~min}$. At 5 min after treatment, cells were fixed with $10 \%$ formalin, stained with the antibody against phosphatidylinositol3,4,5,-triphosphate (PIP3) and DAPI, and visualized by confocal microscopy. Treatment with MES enhanced the immunoreactivity of PIP3, a second messenger molecule produced by PI-3K activation (Fig. 2A). Furthermore, we observed that Akt phosphorylation induced by MES was suppressed in the presence of the PI-3K inhibitors LY294002 and wortmannin (Figs. 2: B and C). These results suggested that MES activates Akt through PI$3 \mathrm{~K}$.

It is well known that activation of the insulin signaling pathway enhances glucose uptake through translocation of GLUT4 in muscle cells. To confirm the effect of MES on insulin signaling, we examined the translocation of GLUT4 in L6 cells. At 5 min after MES treatment, cells were fixed with $10 \%$ formalin, stained with anti-GLUT4 antibody, and visualized by confocal microscopy. Consistent with the activation of the PI-3K/Akt pathway, translocation of intracellular GLUT4 to the plasma membrane was induced by MES (Fig. 3A). We next evaluated the effect of MES on glucose uptake. Differentiated L6 cells were incubated in serum- and glucose-free DMEM medium supplemented with 5\% glutamate for 4 $\mathrm{h}$ and treated with MES for $10 \mathrm{~min}$. At $1 \mathrm{~h}$ after treatment, cells were incubated in serum- and glucose-free DMEM containing $0.5 \mu \mathrm{Ci} / \mathrm{ml} 2$-deoxy-D- $\left({ }^{3} \mathrm{H}\right)$-glucose and $10 \mu \mathrm{M}$ 2-deoxy-D-glucose at $37^{\circ} \mathrm{C}$ for $30 \mathrm{~min}$. After incubation, cells were solubilized with $0.3 \mathrm{M} \mathrm{NaOH}$ and lysates were subjected to liquid scintillation counting. Consistent with the cell surface translocation of GLUT4, glucose uptake was enhanced by MES treatment in L6 cells (Fig. 3B).

Here, we have firstly shown that MES activates the PI-3K/Akt pathway and enhances glucose uptake in 
A.

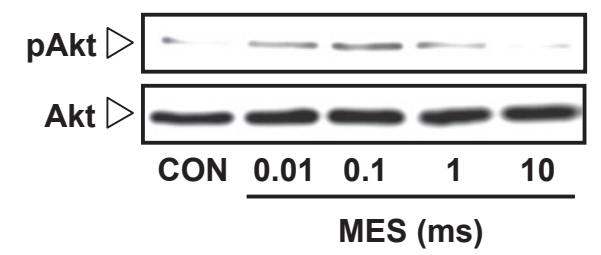

c.

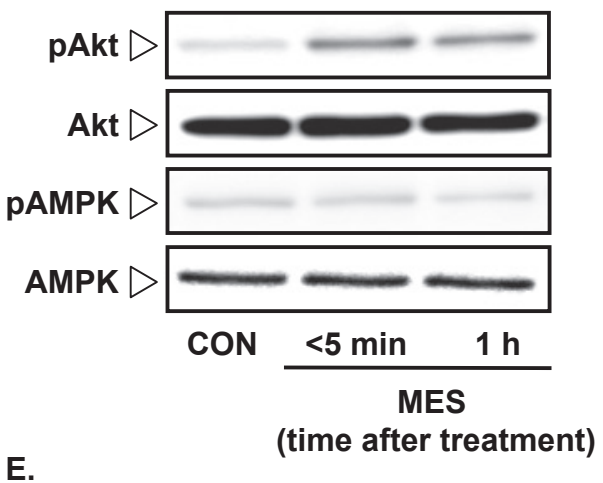

E.

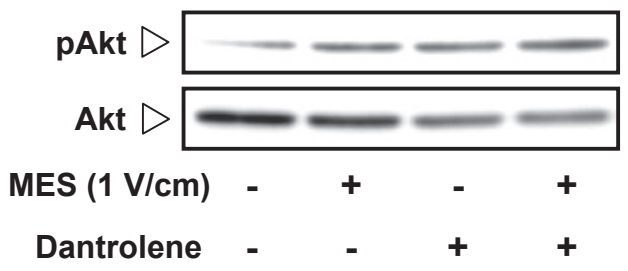

G.

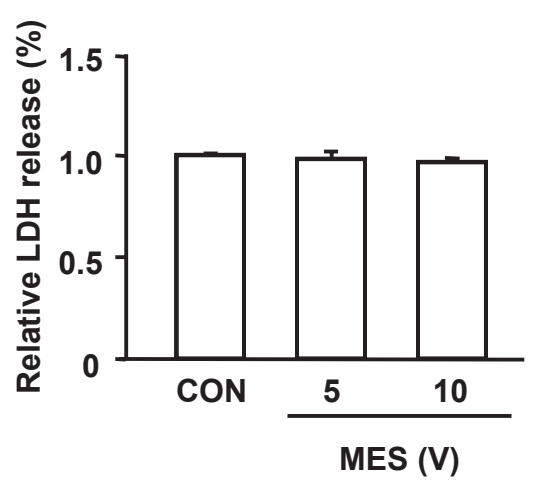

B.

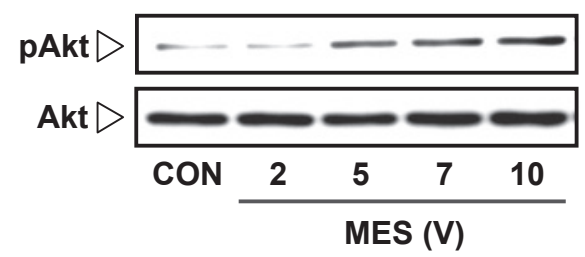

D.

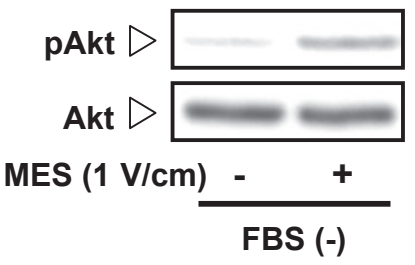

F.



H.



Fig. 1. MES-induced Akt phosphorylation in L6 cells. A, B) Differentiated L6 cells were treated for 10 min with MES at 1 V/ $\mathrm{cm}$ and the indicated millisecond (ms) (A), or at $0.1 \mathrm{~ms}$ and the indicated voltage (B). Cell lysates were immediately extracted after treatment. C) Differentiated cells were treated with MES (1 V/cm, $0.1 \mathrm{~ms}, 55 \mathrm{pps})$ for $10 \mathrm{~min}$. Lysates were extracted immediately $(<5 \mathrm{~min})$ or $1 \mathrm{~h}$ after treatment. D) Differentiated L6 cells were treated with MES (1 V/cm, $0.1 \mathrm{~ms}, 55 \mathrm{pps})$ for $10 \mathrm{~min}$ under serum-free $[\mathrm{FBS}(-)]$ condition. Cell lysates were immediately extracted after treatment. E) Differentiated L6 cells were pre-treated with dantrolene ( $25 \mu \mathrm{M}, 10 \mathrm{~min})$ and then co-treated with MES for $10 \mathrm{~min}$. Lysates were immediately extracted after treatment. F) Differentiated L6 cells were treated with MES (1 V/cm, $0.1 \mathrm{~ms}, 55 \mathrm{pps})$ for $10 \mathrm{~min}$ and/or with the indicated concentration of $\mathrm{KCl}$. Cell lysates were immediately extracted after treatment. For panels A - F, cell lysates were analyzed by Western blotting using the indicated antibodies. G) Lactate dehydrogenase activity was determined in differentiated L6 cells treated for 10 min with MES $(0.1 \mathrm{~ms}, 1$ or $1.5 \mathrm{~V} / \mathrm{cm}, 55 \mathrm{pps})$. \% LDH release was computed as follows: LDH in medium/total LDH. Data are each presented as the mean \pm S.E.M. $(\mathrm{n}=3)$. H) Differentiated L6 cells were treated with MES (1 V/cm, $0.1 \mathrm{~ms}, 55 \mathrm{pps} ; 10 \mathrm{~min})$ or with the mitochondrial respiratory chain inhibitor rotenone $(1 \mu \mathrm{M}, 8 \mathrm{~h})$. Rotenone was used as a positive control. Twenty-four hours after MES treatment, cells were stained with JC-1 and analyzed by flow cytometry. Relative mitochondrial membrane potential $(\Delta \psi \mathrm{m})$ was presented as the mean ratio of JC-1 aggregates $/ \mathrm{JC}-1$ monomer. Data are presented as the mean $\pm \mathrm{S} . \mathrm{E} . \mathrm{M}$. $(\mathrm{n}=3$ per group). ${ }^{* * P}<0.01$, assessed by one-way ANOVA. 
A.

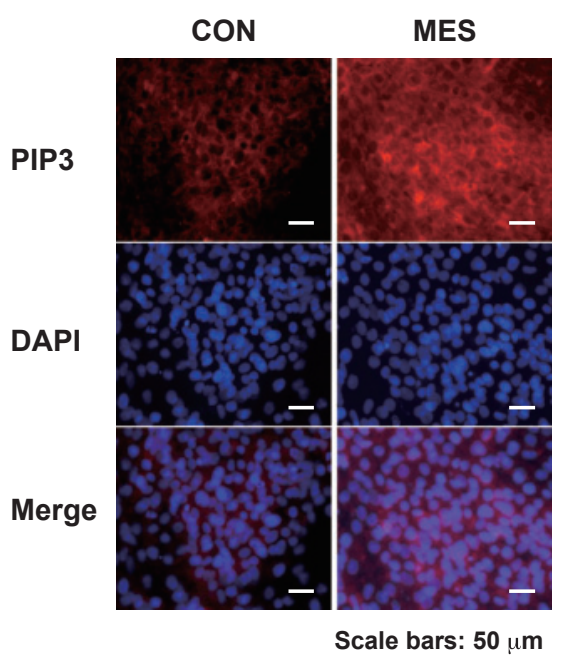

A.

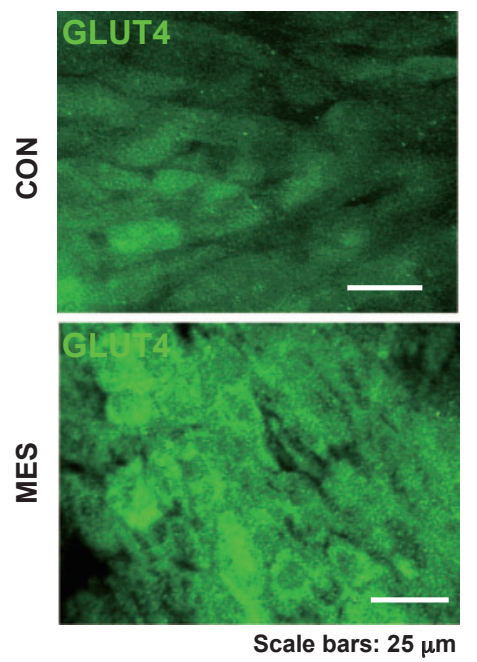

B.

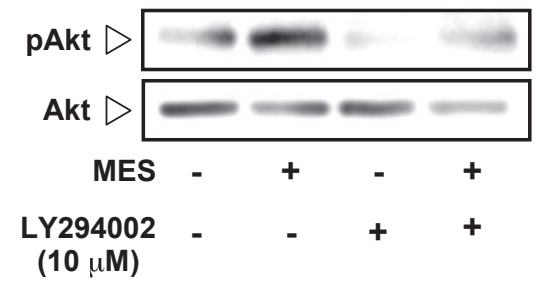

C.

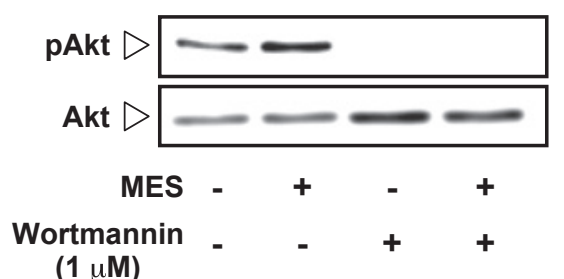

Fig. 2. PI-3K-dependent Akt phosphorylation by MES. A) Differentiated L6 cells were treated with MES (1 V/cm, 0.1 $\mathrm{ms}, 55 \mathrm{pps}$ ) for $10 \mathrm{~min}$, immediately fixed with $10 \%$ formalin, stained with PIP3 antibody and DAPI, and visualized using a confocal fluorescent microscope. B, C) Differentiated L6 cells were treated with MES with or without pre-treatment of LY294002 (10 $\mu \mathrm{M}, 30 \mathrm{~min})$ (B) or wortmannin $(1 \mu \mathrm{M}, 5 \mathrm{~min})(\mathrm{C})$. Cell lysates were immediately extracted after treatment with MES and analyzed by Western blotting with the indicated antibodies.
B.

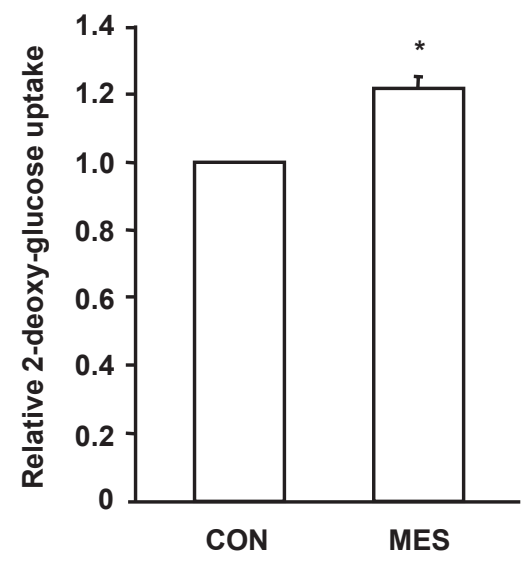

Fig. 3. MES enhanced GLUT4 translocation and glucose uptake in L6 cells. A) Differentiated $\mathrm{L} 6$ cells were treated with MES $(1 \mathrm{~V} / \mathrm{cm}$, $0.1 \mathrm{~ms}, 55 \mathrm{pps}$ ) for $10 \mathrm{~min}$, immediately fixed with $10 \%$ formalin, stained with GLUT4 antibody, and visualized using a confocal fluorescent microscope. B) Differentiated L6 cells were treated with MES $(1 \mathrm{~V} / \mathrm{cm}, 0.1 \mathrm{~ms}, 55$ pps) for $10 \mathrm{~min}$. Then 2-deoxy-D-( $\left.{ }^{3} \mathrm{H}\right)$-glucose was added and cells were subjected to scintillation counting. Data are presented as the mean \pm S.E.M. ( $\mathrm{n}=3$ per group). ${ }^{*} P<0.05$, assessed by one-way ANOVA. muscle cells in the absence of insulin, but exactly how low-intensity electrical current or MES activates PI-3K/ Akt signaling remains unclear. We ruled out the possibility of growth factors because MES could activate Akt in FBS (-) condition (Fig. 1D). Pre-treatment with the muscle relaxant dantrolene also did not affect the Akt activation by MES, although dantrolene alone reduced the total Akt expression and increased Akt phosphorylation (Fig. 1E). This is consistent with a recent study showing that there is a mechanistic difference between in vivo exercise (which causes muscle contraction) and low-intensity electrical current in terms of their effects on elevating the insulin-stimulated glucose transport in skeletal muscle (10). It has been postulated that low-intensity electrical fields can impact on cellular functions by enhancing intracellular signal transduction and that this effect is the basis for the observed therapeutic benefits of applied low-intensity electrical current $(11,12)$. In addition, some studies suggested that physiological electrical current (approximately $1 \mathrm{~V} / \mathrm{cm}$ ) activates integrins $(13,14)$, which leads to the up-regulation of the PI-3K/Akt pathway as a protective cellular response to the electrical signal (15). However, whether the activation of PI-3K/Akt by MES is dependent on integrins remains to be verified. In conclusion, the results presented here describe the enhancing effect of MES on glucose uptake in muscle without insulin and may provide an alternative approach to prevention and amelioration of hyperglycemia. 


\section{Acknowledgments}

This study was supported by a Grant-in-Aid for Scientific Research from the Ministry of Education, Culture, Sports, Science, and Technology (MEXT) of Japan (\#19390045), from the Global COE Program (Cell Fate Regulation Research and Education Unit), MEXT, Japan to H. Kai and from a Grant-in-Aid for JSPS Fellows to S. Yano. The device used for HS + MES treatment was kindly provided by the Tsuchiya Gum Co., Ltd.

\section{References}

1 Taniguchi CM, Emanuelli B, Kahn CR. Critical nodes in signalling pathways: insights into insulin action. Nat Rev Mol Cell Biol. 2006;7:85-96.

2 Gonzalez E, McGraw TE. Insulin signaling diverges into Aktdependent and -independent signals to regulate the recruitment/ docking and the fusion of GLUT4 vesicles to the plasma membrane. Mol Biol Cell. 2006;17:4484-4493.

3 Petersen KF, Shulman GI. Etiology of insulin resistance. Am J Med. 2006;119:S10-S16.

4 Morino S, Kondo T, Sasaki K, Adachi H, Suico MA, Sekimoto E, et al. Mild electrical stimulation with heat shock ameliorates insulin resistance via enhanced insulin signaling. PLoS One. 2008; 3:e4068.

5 Kondo T, Sasaki K, Adachi H, Nakayama Y, Hatemura M, Matsuyama R, et al. Heat shock treatment with mild electrical stimulation safely reduced inflammatory markers in healthy male subjects. Obes Res Clin Pract. 2010;4:e101-e109.

6 Bjornholm M, Zierath JR. Insulin signal transduction in human skeletal muscle: identifying the defects in Type II diabetes. Biochem Soc Trans. 2005;33:354-357.

7 Morino S, Suico MA, Kondo T, Sekimoto E, Yano S, Matsuda T, et al. Mild electrical stimulation increases ubiquitinated proteins and Hsp72 in A549 cells via attenuation of proteasomal degradation. J Pharmacol Sci. 2008;108:222-226.

8 Ito Y, Obara K, Ikeda R, Ishii M, Tanabe Y, Ishikawa T, et al. Passive stretching produces Akt- and MAPK-dependent augmentations of GLUT4 translocation and glucose uptake in skeletal muscles of mice. Pflugers Arch. 2006;451:803-813.

9 Hayashi T, Hirshman MF, Kurth EJ, Winder WW, Goodyear LJ. Evidence for 5' AMP-activated protein kinase mediation of the effect of muscle contraction on glucose transport. Diabetes. 1998;47:1369-1373.

10 Funai K, Schweitzer GG, Castorena CM, Kanzaki M, Cartee GD. In vivo exercise followed by in vitro contraction additively elevates subsequent insulin-stimulated glucose transport by rat skeletal muscle. Am J Physiol Endocrinol Metab. 2010;298: E999-E1010.

11 Seegers JC, Engelbrecht CA, van Papendorp DH. Activation of signal-transduction mechanisms may underlie the therapeutic effects of an applied electric field. Med Hypotheses. 2001;57: 224-230.

12 Robinson KR. The responses of cells to electrical fields: a review. J Cell Biol. 1985;101:2023-2027.

13 Zhao M. Electrical fields in wound healing-An overriding signal that directs cell migration. Semin Cell Dev Biol. 2009;20:674682.

14 Han J, Yan XL, Han QH, Li Y, Zhu J, Hui YN. Electric fields contribute to directed migration of human retinal pigment epithelial cells via interaction between F-actin and beta1 integrin. Curr Eye Res. 2009;34:438-446.

15 Xia H, Nho RS, Kahm J, Kleidon J, Henke CA. Focal adhesion kinase is upstream of phosphatidylinositol 3-kinase/Akt in regulating fibroblast survival in response to contraction of type I collagen matrices via a beta 1 integrin viability signaling pathway. $\mathrm{J}$ Biol Chem. 2004;279:33024-33034. 